\title{
Desarrollo del modelo de negocio canvas en microempresas de las parroquias rurales del Distrito Metropolitano de Quito
}

\section{Development of the canvas business model in micro-enterprises of the rural parishes of the Metropolitan District of Quito}

\author{
Escobar Redín Erika Sofía, \\ Ramírez Terán Víctor Marcelo \\ González Alonso Jesús Francisco \\ Donoso Vargas Diego José \\ Universidad Tecnológica Israel, Ecuador
}

Autor para correspondencia: eescobar@uisrael.edu.ec; mramirez@uisrael.edu.ec Fecha de recepción: 15 de diciembre de 2017 - Fecha de aceptación: 26 de febrero 2018

Resumen: Canvas como propuesta de modelo de negocio constituye una metodología ágil y práctica para estructurar propuestas de valor. Su ámbito de aplicación es amplio: desde las grandes empresas hasta microempresas y emprendimientos. En este trabajo se investigó la aplicación del modelo en seis microempresas pertenecientes a dos parroquiales rurales de Quito: Tababela y Checa. Se trabajó con una muestra aleatoria de 479 clientes de los negocios. El proceso seguido fue una caracterización de cada parroquia, identificación de las microempresas, se aplicó una encuesta a los clientes y una entrevista a los propietarios de los negocios. Con estos insumos se realizó un diagnóstico situacional que implicó los nueve módulos del modelo de negocio Canvas. Estos resultados tributaron al análisis interno y permitieron la elaboración del lienzo para cada negocio. En conclusión, el modelo de negocio Canvas aplicado en las seis microempresas permitirá mejorar sus ingresos, la atención al cliente y consolidarlas como iniciativas empresariales.

Palabras clave: modelo de negocio canvas; microempresa; parroquia rural; Checa; Tababela

\begin{abstract}
Canvas as a business model proposal is an agile and practical methodology for structuring proposals of value. Its scope of application is broad: from large companies to microenterprises and middle enterprises. This paper, describes the application of the model investigated in six microenterprises belonging to two parish councils in Quito: Tababela and Checa. We worked with a random sample of 479 business clients. The process followed was a characterization of each parish, identification of the microenterprises, a survey was applied to the clients and an interview with the owners of the businesses. With these inputs, a situational diagnosis was made involving the nine modules of the Canvas business model. These results were taxed to the internal analysis and allowed the preparation of the canvas for each business. In conclusion, the Canvas business model applied in the six microenterprises will improve their income, customer service and consolidate them as business initiatives.
\end{abstract}

Key words: business model canvas; microenterprise; rural parish; Checa; Tababela 


\section{Introducción}

Hoy, en un mercado donde la globalización es un efecto paradigma, el modelo de negocio Canvas se ha convertido en una herramienta estrella en la gestión estratégica y empresarial de un negocio.

El modelo Canvas mediante el empleo de su lienzo puede detallar de forma gráfica desde la idea de negocio hasta los diferentes factores que influyen al momento de ponerla en acción. A través de nueve módulos que cubren los ámbitos de clientes, oferta, estructura y viabilidad económica, se engloba todo lo necesario para identificar de forma completa la estructura de un negocio.

Las nuevas tendencias del emprendimiento y creación de microempresas en las parroquias de Tababela y Checa del Distrito Metropolitano de Quito (Ecuador) han crecido en los últimos años y han alcanzado distintos mercados.

Canvas es un modelo que pretende explicar cómo se relacionan las distintas áreas del negocio: considerando la segmentación del mercado, teniendo los socios adecuados, recursos y actividades clave, para de esta manera conocer la propuesta de valor que se ofrece a los clientes, captando más consumidores y mejorando la rentabilidad del negocio. Y se lo concibe como un proceso que va desde la identificación de los segmentos de clientes hasta la estructura de costos del negocio (Santandreu Mascarell, Canós Darós y Marín Roig, 2014).

Alexander Osterwalder, especialista en negocios suizo, clarificó el origen y evolución del concepto modelo de negocio (Osterwalder, Pigneur y Tucci, 2005). La cúspide de su aporte la alcanza con la creación del modelo de negocio Canvas cuyo propósito puede expresarse en los siguientes términos:

Establecer una relación lógica entre cada uno de los componentes de la organización y todos los factores que influyen para que tenga o no éxito. Mediante un lienzo "gráfico" se detalla desde la idea de negocios, hasta los diferentes factores que influirán en ella al momento de ponerla en marcha, algunos de ellos que pueden servir como ejemplos para estructurarla eficazmente son: la relación de la marca o producto con su mercado meta, los ingresos y egresos que influyen en su funcionamiento y los factores directos o indirectos que podrían mermar su funcionamiento. (IESDE-Instituto de Estudios Superiores en Dirección de Empresas, 2012, párr. 2).

El minimalismo y la versatilidad del modelo de negocio Canvas son elocuentes, pues como lo expresa Cirugeda (2015):

El modelo Canvas permite ver y moldear en un solo folio, estructurado en nueve elementos, cual es el modelo de nuestro negocio. Y lo mejor de todo, es tan sencillo que puede ser aplicado en cualquier escenario, ya sea una pequeña, mediana y gran empresa. Además, no sólo sirve para las nuevas empresas sino también para aquellas que ya están establecidas. (párr. 2). 
Además, esta herramienta ha sido utilizada en contextos tan diversos como en agencias creativas digitales (Dourado Silva, 2015), análisis de la figura del gestor de eventos (Bernués Oliván, 2015), aplicada a un emprendimiento para posicionar a las mujeres como conductoras de taxi (Macías, Díaz y García, 2015) e incluso como una adaptación para emprendimientos digitales en comunicación (Montalvo-Castro, 2016).

Entre los beneficios de este modelo están que es fácil y sencillo de interactuar, permite trabajar en equipo y a la vez es visual, ya que se puede ver de manera global todos los aspectos importantes que configuran el negocio. Además, es posible aplicarlo a la marca personal y a la carrera profesional (Clark, Osterwalder y Pigneur, 2012).

\section{Métodos}

Este estudio cuantitativo es exploratorio de acuerdo a los alcances de investigación que proponen Hernández, Fernández y Baptista (2014) por cuanto es un trabajo pionero en la aplicación del modelo de negocio Canvas en microempresas de parroquias rurales en Ecuador. En cuanto a tipología de investigación se trata de un estudio documental, de campo y transversal.

La caracterización de las dos parroquias que integraron el estudio (Tababela y Checa) se realizó mediante la técnica del análisis documental sobre fuentes bibliográficas, especialmente los sitios web de las parroquias.

Para diagnosticar la situación de los seis negocios se seleccionó una muestra aleatoria de 479 clientes tal como se describe en la tabla 1.

Tabla 1. Distribución de la muestra de clientes según microempresa

\begin{tabular}{llr}
\hline \multirow{2}{*}{ Parroquias } & \multicolumn{1}{c}{ Microempresas } & Muestras de clientes \\
\hline \multirow{3}{*}{ Tababela } & Restaurante Bachita & 94 \\
& Centro de pesca deportiva El Higuerón & 82 \\
& Panadería y pastelería Adriano Palta & 60 \\
& Tienda de productos alimenticios víveres Ruíz & 95 \\
\multirow{2}{*}{ Checa } & Ciber Dayana & 68 \\
& Feria de productos y servicios mercado Checa ASOMERCHE & 80 \\
& \multicolumn{2}{c}{ Total } \\
\hline
\end{tabular}

Nota: elaboración propia.

A partir de la aplicación de herramientas como la encuesta dirigida a los clientes, las entrevistas a los propietarios y el diagnóstico para determinar las fortalezas y debilidades de los negocios, se aportó información que contribuyó significativamente a la evaluación de los emprendimientos. 
La propuesta de este estudio es el lienzo del modelo de negocio diseñado para cada uno de los seis negocios, atendiendo a los resultados del diagnóstico y a una visión prospectiva del negocio en virtud de lograr su sostenibilidad.

\section{Resultados}

Los resultados de la investigación constituyen, por una parte, el diagnóstico de cada uno de las seis microempresas en términos de sus fortalezas y debilidades así como del estado de sus áreas de gestión: clientes, oferta, estructura y viabilidad económica. Por otra, el lienzo o Canvas de cada uno de los negocios: tres de la parroquia Tababela (Flores Barros, 2017a; Flores Barros, 2017b; Castro Oramas, 2017) y tres de la parroquia Checa (Cevallos Alcívar, 2017; López López, 2017; Toapanta Cupacán, 2017).

En cada lienzo se visualizan, en forma integral, los nueve módulos que caracterizan el modelo de negocio de la microempresa: segmentos de clientes, propuesta de valor, canales, relaciones con los clientes, flujo de ingresos, recursos clave, actividades clave, socios clave y estructura de costos. Como muestra se presentan, en las figuras 1 y 2 , dos lienzos: uno de un negocio de la parroquia Tababela y uno de la parroquia Checa

\section{Conclusiones}

Se puede determinar que en la actualidad el modelo de negocio Canvas es muy utilizado en empresas de todo tipo y dimensión. Esta herramienta de gestión permite, de forma fácil, interactuar con todas las personas que participan dentro del modelo de negocio para llegar a la toma de decisiones.

Con el diagnóstico de la situación de las microempresas y considerando la percepción de los clientes se evidencia que la atención en los negocios, en su gran mayoría, es muy buena. No obstante, en la mayoría de las microempresas se presentan debilidades como la insuficiente infraestructura, no cuentan con una diversificación del negocio y no publican la oferta del negocio en la web y redes sociales.

La aplicación del modelo de negocio Canvas permitió conocer el segmento de clientes al que está dirigido cada negocio, los recursos y actividades clave que se deben utilizar en lo económico, humano e intelectual, con una comunicación constante con los clientes y una correcta interrelación, el manejo adecuado de los costos físicos e intelectuales y generar una apropiada propuesta de valor al cliente.

\section{Agradecimiento}

Los autores agradecen a la Universidad Tecnológica Israel por el apoyo para el desarrollo de la presente investigación. También al Consejo Nacional de Gobiernos Parroquiales Rurales del Ecuador, CONAGOPARE, por la apertura y compromiso con el proyecto y con sus comunidades. Especial agradecimiento a los estudiantes, ahora profesionales, que realizaron el levantamiento de la información de campo en las parroquias que participaron en el estudio. 


\section{Bibliografía}

Baquero, H. et al. (2012). Plan de desarrollo y ordenamiento territorial de la parroquia Tababela 2012-2025. Recuperado de http://www.pichincha.gob.ec/phocadownload/ leytransparencia/literal_k/ppot/dmq/ppdot_tababela.pdf

BernuésOliván, J. (2015). Análisis de la figura del gestor de eventos a través de un modelo de gestión integral: el modelo Canvas(Tesis doctoral, Universidad Complutense de Madrid). Recuperada de http://eprints.ucm.es/37969/1/T37331.pdf

Castro Oramas, G. (2017). Desarrollo del modelo de negocio Canvas en la panadería Adriano Palta perteneciente a la parroquia Tababela (Trabajo de titulación). Universidad Tecnológica Israel, Quito, Ecuador.

Cevallos Alcívar, J. (2017). Desarrollo del modelo de negocio Canvas en el Ciber Dayana perteneciente a la parroquia Checa (Trabajo de titulación). Universidad Tecnológica Israel, Quito, Ecuador.

Cirugeda, E. (2015). ¿Qué es el modelo Canvas? Recuperado de https://www.rankia.com/blog/autonomos-y-emprendedores/2766918-que-modelo-canvas

Clark, T., Osterwalder, A. y Pigneur, Y. (2012).Tu modelo de negocio. Barcelona, España: Deusto.

Dourado Silva, D. (2015). Los modelos de negocios de las agencias creativas digitales de Brasil y España: un estudio exploratorio basado en la metodología Business ModelCanvas(Tesis doctoral, Universidad Carlos III de Madrid). Recuperada de https://earchivo.uc3m.es/handle/10016/22382

Flores Barros, A. M. (2017a). Desarrollo del modelo de negocio Canvas en el restaurante Bachita perteneciente a la parroquia Tababela (Trabajo de titulación). Universidad Tecnológica Israel, Quito, Ecuador.

Flores Barros, D. (2017b). Desarrollo del modelo de negocio Canvas en la pesca deportiva El Higuerón perteneciente a la parroquia Tababela (Trabajo de titulación). Universidad Tecnológica Israel, Quito, Ecuador.

Gobierno Autónomo Descentralizado (GAD) Parroquia Rural de Tababela. (2014). Reseña histórica de la parroquia. Recuperado de http://www.tababela.gob.ec/parroquia.html

Hernández, R., Fernández, C y Baptista, M. P. (2014). Metodología de la investigación (6.a ed.). México D.F.: McGRAW-HILL.

IESDE-Instituto de Estudios Superiores en Dirección de Empresas. (2012). El Modelo Canvas de Alexander Osterwalder. Recuperado de http://iesde.blogspot.com/2012/11/elmodelocanvas-de-alexander.html 
López López, F. (2017). Desarrollo del modelo de negocio Canvas en la tienda de productos alimenticios Víveres Ruiz de la parroquia Checa (Trabajo de titulación). Universidad Tecnológica Israel, Quito, Ecuador.

Macías, R., Díaz, M. y García, G. (2015). Cómo crear un modelo de negocio: caso Taxiwoman. Revista Academia y Negocios, 1(2), 105-114. Recuperado de http://www2.udec.cl/ ran/ojs/index.php/ran/article/view/11/63

Montalvo-Castro, J. (2016). Emprendimientos digitales y diseño de modelos de negocio: investigación aplicada en estudiantes de la carrera de Comunicación. Contratexto, 25, 155$170 . \quad$ Recuperado https://revistas.ulima.edu.pe/index.php/contratexto/article/view/656/633

Osterwalder, A. y Pigneur, Y. (2011). Generación de modelos de negocio: un manual para visionarios, revolucionarios y retadores. Barcelona, España: Deusto.

Osterwalder, O., Pigneur, Y. y Tucci, C.L. (2005). Clarifying Business Models: origins, present, and future of the concept. Communications of the Association for Information Systems, $16(1), 1-25$.

Santandreu Mascarell, C., Canós Darós, L. y Marín Roig, J. R. (2014). Business Model Canvas y redacción del plan de negocio. Universidad Politécnica de Valencia. Recuperado de http://hdl.handle.net/10251/38381

Toapanta Cupacán, C. (2017). Desarrollo del modelo de negocio Canvas para la Feria de Productos y Servicios de la Asociación Mercado de Checa (ASOMERCHE) (Trabajo de titulación). Universidad Tecnológica Israel, Quito, Ecuador. 